\title{
Throughput Analysis of Opportunistic Scheduling under Rayleigh Fading Environment
}

\author{
Erwu Liu and Kin K. Leung \\ Department of Electrical and Electronic Engineering \\ Imperial College, London \\ Exhibition Road, London, SW7 2BT, United Kingdom \\ \{erwu.liu, kin.leung@imperial.ac.uk\}
}

\begin{abstract}
We investigate the proportional fair scheduling (PFS) algorithm, with the objective of obtaining an analytic expression for it. In this paper, we derive a closed-form expression for the throughput of PFS in wireless networks. The theoretical results from analysis are compared with those from simulations. The analytic model is shown to provide a high accuracy in evaluating the throughput of the PFS algorithm in Rayleigh fading environment.
\end{abstract}

Index Terms-proportional fair scheduling, analytic expression, rayleigh fading

\section{INTRODUCTION}

Works have shown that the scheduling policy can significantly affect system performances such as throughput, delay, jitter, fairness and loss rate in wired and wireless networks [1]. Different from wired ones, scheduling in wireless networks needs to consider the unique characteristics of time-varying and location-dependent channel status. Among various related researches on wireless scheduling, the proportional fair scheduling (PFS) algorithm is an attractive solution providing good balance between the maximum throughput and node fairness in a fading environment. In his works on the bandwidth sharing among the elastic flows [2], Kelly derived a rate-allocation vector maximizing the sum of user utility that is known as the PF bandwidth allocation. Since Kelly introduced the concept of PFS, the PFS algorithm in wireless communication networks has garnered much attention [3]-[6] and currently implemented in $3 \mathrm{G}$ wireless for high data rate delay-tolerant services [7].It is known that a $\mathrm{PF}$ problem is NP-hard and it is also hard to obtain a close-to-optimal solution [8]. Until now, researchers [4][5][6][9] mainly rely on computer simulations to assess the performance of the PFS algorithm. In this paper, we focus on mathematical analysis for the node throughput of PFS.

The remainder of the paper is organized as follows. Section II presents the system model and the problem formulation. The mathematical analysis is conducted in Section III: An analytic model is derived for the throughput of PFS under Rayleigh fading environments. In Section IV, numerical and simulation results are presented and compared to validate the closed-form expression given in Section III. The paper is concluded in Section V.

\section{System Model AND Problem Formulation}

For a wireless network shown in Fig. 1, we focus on the link scheduling for node 0 which has radio link connections to its neighboring nodes $m_{l}, m_{2}, \ldots$, and $m_{N}$. Traffic is collected at node 0 before forwarding to the wired Internet.

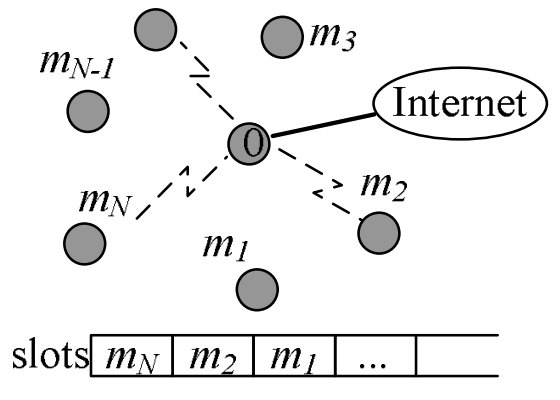

Fig.1. Slot-based scheduling in wireless network

Consider the problem where these nodes $\left(m_{1}, m_{2}, \ldots\right.$, and $\left.m_{N}\right)$ wishing to transmit data in the network (i.e., uplink transmission), and the rates of transmission are randomly varying due to channel fluctuations. Time is divided into small scheduling intervals called slots. In next scheduling slot, the system will estimate the rates by estimating signal to interference-plus-noise ratio (SINR), by use of a pilot signal broadcasted periodically, with a very short delay. The selection of the node to schedule is based on a balance between the current possible rates and fairness. The PFS algorithm performs this by comparing the ratio of the feasible rate for each node to its average throughput tracked by an exponential moving average, which is defined as the preference metric. The node with the maximum preference metric will be selected for transmission at the next scheduling slot. The PFS criteria can be 
described formally as follows. The end of slot $n$ is called time $n$. In next time slot $n+1$, the instantaneous data rate of node $j$ will be $R_{j}[n+1]$. Its $k$-point moving average throughput up to time $n$ is denoted by $r_{j ; k}[n]$, and the preference metric (PF metric) by $M_{j ; k}[n+1]=R_{j}[n+1] / r_{j ; k}[n]$. By definition, the $k$-point moving average $r_{j ; k}[n]$ is defined as the average of its values in last $k$ slots, $r_{j ; k}[m](m=n-k, n-k+1, \ldots n-1)$.

Node $i=\arg \max _{j} M_{j ; k}[n+1]=\arg \max _{j} R_{j}[n+1] / r_{j ; k}[n]$ is chosen to transmit in next time slot $n+1$. The moving average throughput of node $j$ up to time $n+1$ of is updated by

$$
r_{j ; k}[n+1]=\left(1-\frac{1}{k}\right) r_{j ; k}[n]+I_{j}[n+1] \times \frac{R_{j}[n+1]}{k}
$$

where $I_{j}[n+1]$ is the indicator function of the event that node $j$ is scheduled to transmit in time slot $n+1$.

$I_{j}[n+1]= \begin{cases}1, & \text { node } j \text { scheduled in slot } n+1 \\ 0, & \text { else }\end{cases}$

By introducing user utility function $U_{j}=\log \left[r_{j}\right]$, Kelly [2] had proved that the sum of the user utility (user satisfaction indicator) is maximized under the PFS criteria. Obviously, for a time-varying fading environment, when the node number is large, there will always be high probability that some nodes are in the good channel status. On the other hand, the PFS provides some sense of fairness, in that nodes frequently in the bad channel status have low throughput which in turn tends to increase their probability of being scheduled. It is the logarithm utility maximization characteristics, the multi-user diversity gain and the possibility to schedule bad-channel-condition nodes that make the PF scheduler superior to the traditional ones such as round-robin (RR) and opportunistic scheduler.

Though the PFS algorithm described by (1) and (2) has fairly low implementation complexity in that each scheduling slot requires only $N$ addition operations and $2 N+1$ multiplication operations for given $N$ nodes, it is in fact NP-hard to obtain its analytic solution [8]. Basically, there are two models commonly used to simplify the analysis of PFS: Linear model and logarithm model [10][11]. In the linear model, the feasible rate is linearly proportional to the SINR, while in the logarithm model, there is a logarithmic relationship between the SINR and the feasible rate. Different from these two common-used rate models, P. J. Smith [12] states that in a Rayleigh fading environment, the feasible rate $R$ could be approximated by a Gaussian distribution with surprisingly high accuracy. For single-input-single-output (SISO) case, it reduces to

$$
\begin{gathered}
E[R]=\int_{0}^{\infty} \log (1+\operatorname{SINR} \times \lambda) \times e^{-\lambda} d \lambda \\
\sigma_{R}^{2}=\int_{0}^{\infty}(\log (1+\operatorname{SINR} \times \lambda))^{2} \times e^{-\lambda} d \lambda \\
-\left(\int_{0}^{\infty} \log (1+\operatorname{SINR} \times \lambda) \times e^{-\lambda} d \lambda\right)^{2}
\end{gathered}
$$

where $E[R]$ and $\sigma_{R}$ are the mean value and the standard deviation of $R$.

As PF metric is directly related to the feasible rate $R$, for our analysis to be as accurate as possible, it is natural for us to assume in our analysis that the feasible rate over Rayleigh fading channels is Gaussian [12].

\section{Closed-Form EXPRESSION FOR PFS ThroughPUT}

From (1), assuming wide-sense stationary $r_{j}$, the expected value of the $k$-point moving average throughput of node $j$ up to time $n+1$ is

$$
\left.\left.E \mid r_{j ; k}[n+1]\right]=E \mid r_{j ; k}[n]\right]=E\left[I_{j}[n+1] R_{j}[n+1]\right](5)
$$

Applying (2) to (5) yields

$$
\begin{aligned}
\left.E \mid r_{j ; k}[n]\right] & =E\left[I_{j}[n+1] R_{j}[n+1]\right] \\
& =E\left[R_{j}[n+1] \mid I_{j}[n+1]=1\right] \times \operatorname{Pr}\left(I_{j}[n+1]=1\right)
\end{aligned}
$$

where $\operatorname{Pr}\left(I_{j}[n+1]=1\right)$ is the average probability that node $j$ will be scheduled in time slot $n+1$.

With Bayes's theorem, we can write (6) as

$$
E\left[r_{j ; k}[n]\right]=\int_{0}^{\infty} x f_{R_{j}}(x) \operatorname{Pr}\left(I_{j}[n+1]=1 \mid R_{j}[n+1]=x\right) d x(7)
$$

where $\operatorname{Pr}\left(I_{j}[n+1]=1 \mid R_{j}[n+1]=x\right)$ is the conditional probability that node $j$ will be scheduled in time slot $n+1$, given that the feasible rate of node $j$ in time slot $n+1$ took on the value $x, f_{R j}($. the probability density function of $R_{j}$.

Under the PFS criteria presented in Section II, for statistically independent $R_{j}$ and wide-sense stationary, first-order ergodic $r_{j ; k}$ , it holds for large $n, k$ that

$$
\begin{aligned}
\operatorname{Pr}\left(I_{j}[n+1]=1 \mid R_{j}[n+1]=x\right) & =\prod_{i=1, i \neq j}^{N} F_{R_{i}}\left(\frac{x}{r_{j ; k}[n]} r_{i ; k}[n]\right) \\
& \approx \prod_{i=1, i \neq j}^{N} F_{R_{i}}\left(\frac{E\left[r_{i}\right]}{E\left[r_{j}\right]} x\right)
\end{aligned}
$$

where $r_{j ; k}[n]$ is the $k$-point moving average throughput of node $j$ up to time $n, F_{R j}($.$) the distribution function of R_{j}$.

For Gaussian distribution $R_{j}$ as described in Section II, on substitution of (8) into (7), we obtain

$$
\begin{aligned}
E\left[r_{j}\right] & =\int_{0}^{\infty} x f_{R_{j}}(x) \prod_{i=1, i \neq j}^{N} F_{R_{i}}\left(\frac{E\left[r_{i}\right]}{E\left[r_{j}\right]} x\right) d x \\
& =\int_{-\frac{E\left[R_{j}\right]}{\sigma_{R_{j}}}}^{\infty}\left(y \sigma_{R_{j}}+E\left[R_{j}\right]\right) \frac{1}{\sqrt{2 \pi}} e^{-\frac{y^{2}}{2}} \\
& \times \prod_{i=1, i \neq j}^{N} F_{R_{i}}\left(\frac{E\left[r_{i}\right]}{E\left[r_{j}\right]}\left(y \sigma_{R_{j}}+E\left[R_{j}\right]\right)\right) d y
\end{aligned}
$$

where $E\left[R_{j}\right], \sigma_{R j}$ are the expect value and standard deviation of $R_{j}$, respectively.

From (3) and (4), one can prove that $\sigma_{R j}>\sigma_{R i}$ and $E\left[R_{j}\right] / \sigma_{R j}>E\left[R_{i}\right] / \sigma_{R i}$ if $E\left[R_{j}\right]>E\left[R_{i}\right]$. Obviously, with proportional fair scheduling, node $j$ will have higher mean throughput $E\left[r_{j}\right]$ for higher $E\left[R_{j}\right]$ and $\sigma_{j}$. We then have $\left(E\left[r_{j}\right] E\left[R_{i}\right]-E\left[r_{i}\right] E\left[R_{j}\right]\right) /\left(E\left[r_{i}\right] \sigma_{R j}-E\left[r_{j}\right] \sigma_{R i}\right)<0$ for all $\sigma_{R j} \neq \sigma_{R i}$. So when all $\sigma_{R i}(i=1,2, \ldots, N)$ are equal, with the fact $F_{R i}(x)=F_{(0,1)}\left(\left(x-E\left[R_{i}\right]\right) / \sigma_{R i}\right)$ for Gaussian $R_{i}$, where $F_{0,1}($.$) is zero$ 
mean, unit variance standard normal distribution function

$$
\begin{aligned}
E\left[r_{j}\right] & =\int_{-\frac{E\left[R_{j}\right]}{\sigma_{R_{j}}}}^{\infty}\left(y \sigma_{R_{j}}+E\left[R_{j}\right]\right) \frac{1}{\sqrt{2 \pi}} e^{-\frac{y^{2}}{2}} \\
& \times \prod_{i=1, i \neq j}^{N} F_{R_{i}}\left(\frac{E\left[r_{i}\right]}{E\left[r_{j}\right]}\left(y \sigma_{R_{j}}+E\left[R_{j}\right]\right)\right) d y \\
& =\int_{-\frac{E\left[R_{j}\right]}{\sigma_{R_{j}}}}^{\infty}\left(y \sigma_{R_{j}}+E\left[R_{j}\right]\right) \frac{1}{\sqrt{2 \pi}} e^{-\frac{y^{2}}{2}} \\
& \times\left(F_{R_{j}}\left(y \sigma_{R_{j}}+E\left[R_{j}\right]\right)\right)^{N-1} d y \\
& =\int_{-\frac{E\left[R_{j}\right]}{\sigma_{R_{j}}}}^{\infty}\left(y \sigma_{R_{j}}+E\left[R_{j}\right]\right) f_{(0,1)}(y) \times\left(F_{(0,1)}(y)\right)^{N-1} d y
\end{aligned}
$$

where $f_{0,1}($.$) is zero mean, unit variance standard normal$ probability density function.

Denote $Z=\arg \max _{i}\left[\left(E\left[r_{j}\right] E\left[R_{i}\right]-E\left[r_{i}\right] E\left[R_{j}\right]\right) /\left(E\left[r_{i}\right] \sigma_{R j}-E\left[r_{j}\right] \sigma_{R i}\right)\right]$, it is easy to verify that $Z \geq\left(E\left[r_{j}\right] E\left[R_{i}\right]-E\left[r_{i}\right] E\left[R_{j}\right]\right) /\left(E\left[r_{i}\right] \sigma_{R j}-E\left[r_{j}\right] \sigma_{R i}\right) \geq-E\left[R_{j}\right] / \sigma_{R j} \quad$ and, $Z \leq-\arg \max _{i}\left[E\left[R_{i}\right] / \sigma_{R i}\right]$. So when not all $\sigma_{R i}$ are equal, (9) can be written as

$$
\begin{aligned}
E\left[r_{j}\right] & =\int_{-\frac{E\left[R_{j}\right]}{\sigma_{R_{j}}}}^{Z}\left(y \sigma_{R_{j}}+E\left[R_{j}\right]\right) f_{(0,1)}(y) \\
& \times \prod_{i=1, i \neq j}^{N} F_{R_{i}}\left(\frac{E\left[r_{i}\right]}{E\left[r_{j}\right]}\left(y \sigma_{R_{j}}+E\left[R_{j}\right]\right)\right) d y+ \\
& \int_{Z}^{\infty}\left(y \sigma_{R_{j}}+E\left[R_{j}\right]\right) f_{(0,1)}(y) \\
& \times \prod_{i=1, i \neq j}^{N} F_{R_{i}}\left(\frac{E\left[r_{i}\right]}{E\left[r_{j}\right]}\left(y \sigma_{R_{j}}+E\left[R_{j}\right]\right)\right) d y
\end{aligned}
$$

As the first integral in the right hand of (11) is not less than 0 , we have

$$
\begin{aligned}
E\left[r_{j}\right] & \geq \int_{Z}^{\infty}\left(y \sigma_{R_{j}}+E\left[R_{j}\right]\right) f_{(0,1)}(y) \\
& \times \prod_{i=1, i \neq j}^{N} F_{R_{i}}\left(\frac{E\left[r_{i}\right]}{E\left[r_{j}\right]}\left(y \sigma_{R_{j}}+E\left[R_{j}\right]\right)\right) d y
\end{aligned}
$$

On the other hand, as $\left(E\left[r_{i}\right] / E\left[r_{j}\right]\right) \times\left(y \sigma_{R j}+E\left[R_{j}\right]\right) \geq\left(y \sigma_{R i}+E\left[R_{i}\right]\right)$ for any $y \geq Z \geq\left(E\left[r_{j}\right] E\left[R_{i}\right]-E\left[r_{i}\right] E\left[R_{j}\right]\right) /\left(E\left[r_{i}\right] \sigma_{R j}-E\left[r_{j}\right] \sigma_{R i}\right)$, we then have

$$
\begin{aligned}
E\left[r_{j}\right] & \geq \int_{Z}^{\infty}\left(y \sigma_{R_{j}}+E\left[R_{j}\right]\right) f_{(0,1)}(y) \\
& \times \prod_{i=1, i \neq j}^{N} F_{R_{i}}\left(y \sigma_{R_{i}}+E\left[R_{i}\right]\right) d y \\
& =\int_{Z}^{\infty}\left(y \sigma_{R_{j}}+E\left[R_{j}\right]\right) f_{(0,1)}(y)\left(F_{(0,1)}(y)\right)^{N-1} d y \\
& \geq \int_{M}^{\infty}\left(y \sigma_{R_{j}}+E\left[R_{j}\right]\right) f_{(0,1)}(y)\left(F_{(0,1)}(y)\right)^{N-1} d y
\end{aligned}
$$

wher $M=-\arg \max _{i}\left[E\left[R_{i}\right] / \sigma_{R i}\right](i=1,2, \ldots, N)$.

Obviously, (10) and (13) can be described by the same expression,

$$
\begin{aligned}
E\left[r_{j}\right] & \geq \int_{M}^{\infty}\left(y \sigma_{R_{j}}+E\left[R_{j}\right]\right) f_{(0,1)}(y)\left(F_{(0,1)}(y)\right)^{N-1} d y \\
& \approx \frac{E\left[R_{j}\right]}{N}+\int_{M}^{\infty} y \sigma_{R_{j}} f_{(0,1)}(y)\left(F_{(0,1)}(y)\right)^{N-1} d y
\end{aligned}
$$

We now have the closed-form expression for the mean throughput of a node in the PFS-capable network.

(14) states that PFS will benefit more in severe fading environments where $\sigma_{R j}$ is large. On the other hand, PFS can be viewed as RR scheduling in no-fading environments where $\sigma_{R j}$ is very small. In addition, (14) reveals a very interesting merit of PFS that has not been seen elsewhere in literatures: node $j$ 's throughput is solely determined by the network size together with the first order statistics of its own feasible rate.

One would notice that most theoretical results in literatures [10] [11] are obtained with the assumption that there are some kinds of i.i.d relationship among nodes. For example, [11] assumes that node $k$ 's SINR $S_{k}=c_{k} \times C(\forall k)$, where $c_{k}$ is a node-related constant and $C$ is a distribution independent of all nodes (i.e., $C$ is i.i.d for all nodes). Clearly, our theoretical results are more general as we do not rely on the i.i.d assumption in the derivation.

\section{Numerical and Simulation Results}

We now evaluate the validity of the expression given by (14) under various scenarios.

In the simulation, the feasible rates over Rayleigh fading channels are generated according to statically independent Gaussian distribution for different nodes and the initial moving average throughputs of nodes are randomized. System parameters are: $20 \mathrm{MHz}$ bandwidth, $k=500$ for $k$-point moving average calculation.

For simplicity, we use the notation $n_{j}\left[E\left[R_{j}\right], \sigma_{R j}\right]$ to indicate the feasible rate of node $j$ has mean value $E\left[R_{j}\right]$ and standard deviation $\sigma_{R j}$.

\section{Scenario A:}

For any $j$, node $j$ has randomized data rate mean $E\left[R_{j}\right]$ and small standard deviation $\sigma_{R j}$. The feasible data rates (in Mbps) of nodes are characterized as:

$n_{1}[80,0.6], \quad n_{2}[40,0.2], \quad n_{3}[22,0.1], \quad n_{4}[90,0.8], \quad n_{5}[35,0.3]$, 
$n_{6}[32,0.2], \quad n_{7}[25,0.2], \quad n_{8}[38,0.4], \quad n_{9}[18,0.3], \quad n_{10}[20,0.3]$

This case corresponds to the scenario where the fading effect could be neglected. One can verify that if we apply round robin (RR) scheduler to the nodes as above, node 1 will have a throughput of about $80 / 10=8.0$ Mbps. The accuracy of (14) is shown in Fig. 2. As stated earlier in Section III, both numerical and simulation results in Fig. 2 indicate the PFS algorithm is in fact a RR scheduling algorithm in this scenario.

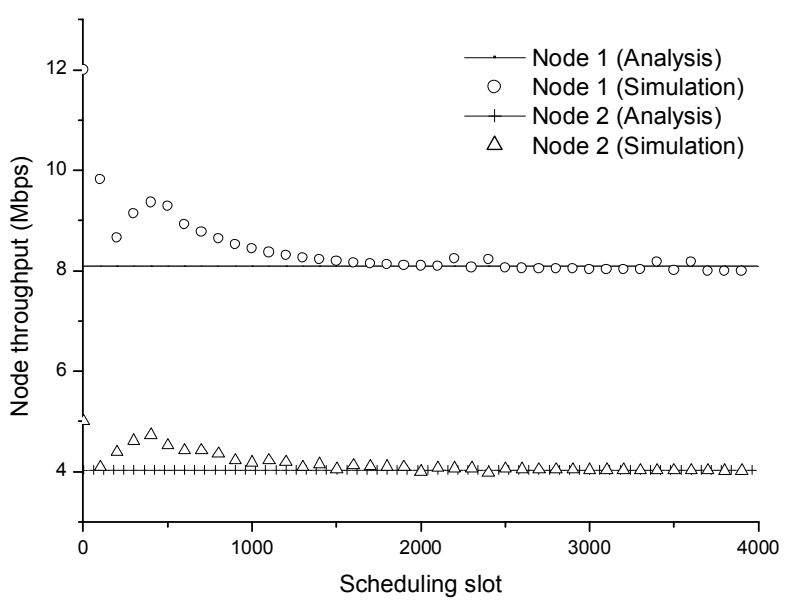

Fig.2. Accuracy of our analytic expression (scenario A)

Scenario B:

For any $j$, node $j$ has randomized data rate mean $E\left[R_{j}\right]$ and proportional standard deviation $\sigma_{R j}$. In fact, most researches [10] [11] use assumptions similar to this scenario in there derivations. For example, [11] assumes that node $k$ 's SINR $S_{k}=c_{k} \times C(\forall k)$, where $c_{k}$ is a node-related constant and $C$ is a distribution independent of all nodes. It is obvious that [11] assumes a proportional relationship between the mean and standard deviation of $\operatorname{SINR} S_{k}$, i.e., $E\left[S_{k}\right] / \sigma_{S k}=E[C] / \sigma_{C}$ is constant for all nodes. One can verify by numerical calculation that our expression given by (14) will give throughput result very close to the existing one given by [11] in this scenario.

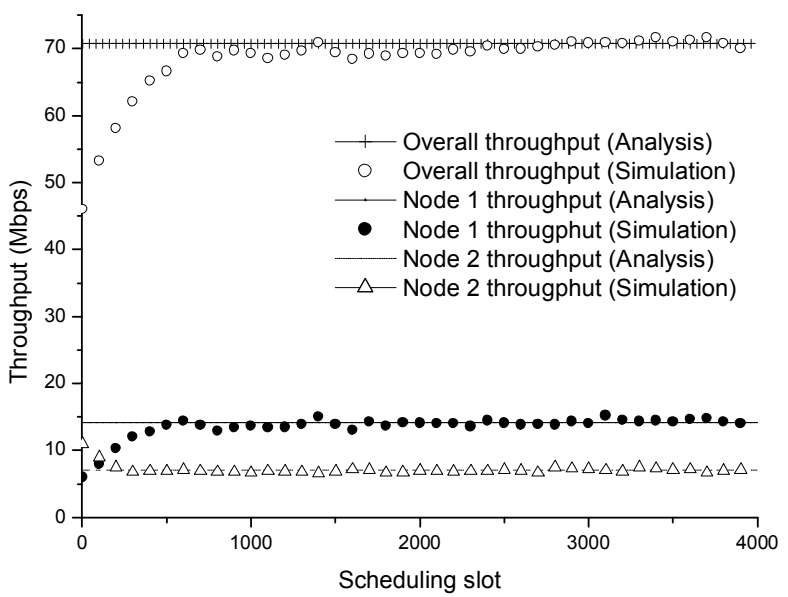

Fig.3. Accuracy of our analytic expression (scenario B)
We then plot in Fig. 3 the throughput from numerical analysis and simulation. The feasible data rates (in Mbps) of nodes are characterized as:

$n_{1}[80,40], \quad n_{2}[40,25], \quad n_{3}[18,9], n_{4}[80,40], \quad n_{5}[35,17.5]$, $n_{6}[28,14], \quad n_{7}[20,10], \quad n_{8}[28,14], n_{9}[18,9], \quad n_{10}[20,10]$ where $\sigma_{R j} / E\left[R_{j}\right]=0.5$ proportional factor is assumed.

As expected, the simulation results in scenario $\mathrm{A}$ and $\mathrm{B}$ indicate a high accuracy of our analytic expression for PFS.

We would like to point out that, in wireless networks, feasible data rate depends largely on user location, channel fading characters, modulation schemes, etc., so the assumption of scenario A and B may not fully reflect the data rate pattern in a real wireless network. For the analysis to be complete, we investigate the validity of (14) in more general cases.

\section{Scenario C:}

For any $j$, node $j$ has randomized data rate mean $E\left[R_{j}\right]$ and standard deviation $\sigma_{R j}$ given by (3) and (4).

To test the analytic expression under different load and SINR, we change the number of nodes from 1 to 50 , i.e., incrementally add in the nodes characterized as follows, with the SINR of node 1 fixed at $0.8 \mathrm{~dB}, 10.8 \mathrm{~dB}$ and $20.8 \mathrm{~dB}$, respectively.

The feasible rates (in mega nats per second, 1 nat $=1.443$ bit) of node 1 are $n_{1}[13.5,9.2], n_{1}[43.3,18.9]$, and $n_{1}[85.1,23.8]$ for $S I N R=0.8 \mathrm{~dB}, 10.8 \mathrm{~dB}$ and $20.8 \mathrm{~dB}$, respectively. The feasible rates of other nodes are randomized as: $n_{2}$ [87.3,24.0], $n_{3}$ [24.1,13.7], $n_{4}$ [34.9,16.9], $n_{5}[129,25.3]$, $n_{6}[91.8,24.2], \quad n_{7}[70.6,22.7], n_{8}[37.4,17.6], n_{9}[150,25.5]$, $n_{10}[27.7,14.9], n_{11}[45.9,19.4], n_{12}[78.0,23.4], n_{13}[34.2,16.8]$, $n_{14}[102,24.6], n_{15}[86.9,23.9], n_{16}[49.9,20.1], n_{17}[25.5,14.2]$, $n_{18}[32.5,16.3], n_{19}[118,25.1], n_{20}[79.4,23.5], n_{21}[141,25.4]$, $n_{22}[27.7,14.9], n_{23}[34.9,16.9], n_{24}[46.3,19.5], n_{25}$ [37.8,17.6], $n_{26}[86.0,23.9], n_{27}[70.6,22.7], n_{28}[77.2,23.3], n_{29}[127,25.2]$, $n_{30}[108,24.8], n_{31}[86.0,23.9], n_{32}[60.0,21.6], n_{33}[133,25.3]$, $n_{34}[27.7,14.9], n_{35}[86.0,23.9], n_{36}[60.0,21.6], n_{37}[47.1,19.6]$, $n_{38}[90.5,24.1], n_{39}[158.9,25.5], n_{40}[182,25.6], n_{41}[64.2,22.1]$, $n_{42}[36.7,17.4], n_{43}[24.4,13.8], n_{43}[34.2,16.8], n_{45}[42.1,18.6]$, $n_{46}[88.2,24.0], n_{47}[65.5,22.2], n_{48}[90.5,24.1], n_{49}[71.9,22.9]$, $n_{50}[91.8,24.2]$

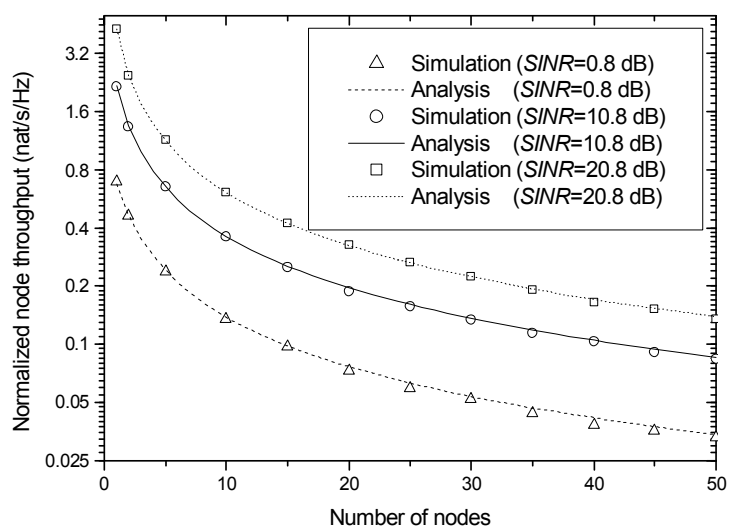

Fig.4. Accuracy of our analytic expression (scenario C) 
We plot in Fig.4 the normalized throughput (nat $/ \mathrm{s} / \mathrm{Hz}$ ) of node 1 obtained from numerical analysis and simulations.

Once again, Fig. 4 justifies the accuracy of equation (14) for different SINR and node number.

The overall conclusion from these experiments is that the closed-form expressions presented in Section III can be used with confidence to evaluate the performance of the PFS algorithm under various Rayleigh fading environments.

\section{CONCLUSIONS AND FutURE WORK}

In this paper, we develop analytic solution for the throughput of proportional fair scheduling in Rayleigh fading environment. It is shown through simulations that our analytic expression provides an accurate estimate of the throughput of PFS. Moreover, our theoretical results are more general than existing ones [10] [11] in that we do not assume i.i.d relationship among nodes in our derivations. The results are being integrated into the MEMBRANE project [13].

Here we only considered the single-link PFS and one-hop network, multi-link PFS algorithms in MIMO antenna equipped networks and multi-hop environments will be the next stage of our research.

\section{ACKNOWLEDGMENT}

This research was financially supported by grant from EU IST FP6 MEMBRANE project under the contract 027310.

\section{REFERENCES}

[1] A. K. Parekh and R. G. Gallager, "A generalized processor sharing approach to flow control in integrated services networks: The single-node case," IEEE/ACM Trans. Netw., vol. 1, no. 3, pp. 344-357, Jun. 1993.

[2] F. Kelly, "Charging and Rate Control for Elastic Traffic", Eur. Trans. on Telecommun., Feb. 1997, pp. 33-37.

[3] J. M. Holtzman, "Asymptotic analysis of proportional fair algorithm," in Proc. IEEE PIMRC, San Diego, CA, 2001, pp. 33-37.

[4] T. D. Nguye and Y. Han, "A Proportional Fairness Algorithm with QoS Provision in Downlink OFDMA Systems," IEEE Commun Lett., vol. 10, no. 11 , Nov. 2006

[5] H. Seo and B. G. Lee, "A Proportional-Fair Power Allocation Scheme for Fair and Efficient Multiuser OFDM Systems," in Proc. IEEE GLOBECOM, Dallas TX, 2004, pp. 3737-3741.

[6] Z. Han, Z. Ji and K. J. Ray Liu, "Fair Multiuser Channel Allocation for OFDMA Networks Using Nash Bargaining Solutions and Coalitions," IEEE Trans. Commun., vol. 53, no. 8, pp. 1366-1376, Aug. 2005.

[7] A. Jalali, R. Padovani and R. Pankai, "Data Throughput of CDMA-HDR: a High Efficiency-High Data Rate Personal Communication Wireless System," in Proc. Veh. Technol. Conf., Jan. 2001, pp. 55-67.

[8] T. Bu, L. Li and R. Ramjee, "Generalized Proportional Fair Scheduling in Third Generation Wireless Data Networks," in Proc. INFOCOM, Barcelona, Apr. 2006, pp. 1-12.

[9] C. Wengerter, J. Ohlhorst and A. G. E. von Elbwart, "Fairness and Throughput Analysis for Generalized Proportional Fair Frequency Scheduling in OFDMA," in Proc. Veh. Technol. Conf., Jun. 2005, pp. 1903-1907.

[10] D. Avidor, S. Mukherjee, J. Ling and C. Papadias, "On Some Properties of the Proportional Fair Scheduling Policy," in Proc. IEEE PIMRC, New Jersey, Sep. 2004, pp. 853-858.

[11] J-G. Choi and S. Bahk, "Cell-Throughput Analysis of the Proportional Fair Scheduler in the Single-Cell Environment," IEEE Trans. Veh. Technol., vol. 56, no. 2, pp. 766-778, Mar. 2007.

[12] P. J. Smith and M. Shafi, "On a Gaussian Approximation to the Capacity of Wireless MIMO Systems," in Proc. IEEE ICC, New York, Apr. 2002, pp. 406-410.

[13] The MEMBRANE website. [Online]. Available: http://www.imperial.ac.uk/MEMBRANE 